\title{
BMJ Open Comparative efficacy and acceptability of psychotherapies for acute anxiety disorders in children and adolescents: study protocol for a network meta- analysis
}

\author{
Yuqing Zhang, ${ }^{1}$ Xinyu Zhou, ${ }^{1}$ Anthony C James, ${ }^{2,3}$ Bin Qin, ${ }^{1}$ Craig J Whittington, ${ }^{4}$ \\ Pim Cuijpers, ${ }^{5}$ Cinzia Del Giovane, ${ }^{6}$ Yiyun Liu, ${ }^{1}$ David Cohen, ${ }^{7}$ John R Weisz, ${ }^{8}$ \\ Peng $\mathrm{Xie}^{1}$
}

To cite: Zhang $Y$, Zhou $X$, James AC, et al. Comparative efficacy and acceptability of psychotherapies for acute anxiety disorders in children and adolescents: study protocol for a network metaanalysis. BMJ Open 2015;5: e008572. doi:10.1136/ bmjopen-2015-008572

- Prepublication history and additional material is available. To view please visit the journal (http://dx.doi.org/ 10.1136/bmjopen-2015008572)

Yuqing Zhang and Xinyu Zhou contributed equally to the protocol.

Received 22 April 2015 Revised 29 August 2015 Accepted 9 September 2015

CrossMark

For numbered affiliations see end of article.

Correspondence to Professor Peng Xie; xiepeng973@126.com

\section{ABSTRACT}

Introduction: Anxiety disorders are associated with significant public health burden in young individuals. Cognitive-behavioural therapy (CBT) is the most commonly used psychotherapy for anxiety disorders in children and adolescents, but previous reviews were hindered by a limited number of trials with direct comparisons between different psychotherapies and their deliveries. Consequently, the main aim of this research was to investigate the comparative efficacy and acceptability of various types and deliveries of psychotherapies for anxiety disorders in children and adolescents.

Methods and analysis: We will systematically search PubMed, EMBASE, Cochrane, Web of Science, PsycINFO, CINAHL, ProQuest Dissertations and LiLACS for randomised controlled trials, regardless of whether participants received blinding or not, published from 1 January 1966 to 30 January 2015 (updated to 1 July 2015), that compared any psychotherapy with either a control condition or an active comparator with different types and/or different delivery formats for the acute treatment of anxiety disorders in children and adolescents. Data extraction, risk of bias and quality assessments will be independently extracted by two reviewers. The primary outcome for efficacy will be mean overall change scores in anxiety symptoms (self-rated or assessor-rated) from baseline to post-treatment between two groups. The acceptability of treatment will be measured as the proportion of patients who discontinued treatment during the acute phase of treatment. We will assess efficacy, based on the standardised mean difference (SMD), and acceptability, based on the OR, using a random-effects network meta-analysis within a Bayesian framework. Subgroup and sensitivity analyses will be conducted to assess the robustness of the findings.

Ethics and dissemination: No ethical issues are foreseen. The results will be published in a peer-reviewed journal and will be disseminated electronically and in print. The meta-analysis may be updated to inform and guide management of anxiety in children and adolescents.

\section{Strengths and limitations of this study}

- Network meta-analysis combines direct and indirect evidence for all relative treatment effects (for the selected outcomes) and provides estimates with maximum power.

- We will comprehensively assess the efficacy and acceptability of psychotherapies for anxiety disorder in children and adolescents at the end of the acute treatment and at follow-up.

- This study will inform clinical decision-making about which of the different types (and/or different delivery formats) of psychotherapies are better to treat children and adolescents suffering from anxiety disorders.

Trial registration number: PROSPERO CRD42015016283.

\section{BACKGROUND}

Anxiety disorders are among the most common mental disorders during childhood and adolescence, with a prevalence of $3-5 \%$ in school-age children (6-12 years) and 10$19 \%$ in adolescents (13-18 years) $;^{12}$ and the prevalence of anxiety disorders in this population tends to increase over time. ${ }^{3}$ Anxiety is the most common psychological symptom reported by children and adolescents; however, presentation varies with age as younger patients often report undifferentiated anxiety symptoms, for example, muscle tension, headache, stomachache or angry outbursts. ${ }^{4}$ According to the standard diagnostic systems, there are various types of anxiety disorders, for example, generalised anxiety disorder (GAD), social phobias (SOP), social 
anxiety disorder (SAD), panic disorder (PD), overanxious disorder, separation anxiety, post-traumatic stress disorder (PTSD), obsessive-compulsive disorder (OCD). ${ }^{5}$ Anxiety disorders in children and adolescents often occur with a number of comorbidities, such as autism spectrum disorders ${ }^{6}$ depressive disorders, ${ }^{7}$ conduct disorder, ${ }^{8}$ substance abuse ${ }^{9}$ or suicide-related behaviour. ${ }^{10}$ Youths with anxiety disorders experience serious impairment in social functioning (eg, poor school achievement; relational problems with family members and peers). ${ }^{11} 12$ Childhood and adolescent anxiety disorders can persist despite treatment, ${ }^{1}$ and they are associated with later adult psychopathology. ${ }^{13} 14$

Psychotherapy and/or medication have been the mainstay of treatment for anxiety disorders in children and adolescents. ${ }^{15}$ Although there appear to be few specific guidelines recommending psychotherapy, it is often preferred as first-line therapy, if it is available, due to the acceptability of psychological treatments. ${ }^{16}{ }^{17}$ The most commonly practised psychological treatment for anxiety disorders in children and adolescents is cognitive-behavioural therapy (CBT). ${ }^{18}$ One of the first CBT programmes for the treatment of anxiety disorders in children and adolescents was 'Coping Cat', which includes components concerned with the identification and modification of negative automatic thoughts, psychoeducation, exposure to feared stimuli, training in coping skills and problem-solving. ${ }^{19}$ Many of the subsequent CBT programmes were influenced by 'Coping Cat'. ${ }^{20}$ Recently, some other psychotherapies, for example, cognitive bias modification (CBM), ${ }^{21}$ have been developed and evaluated for the treatment of anxiety disorders in children and adolescents.

Despite the high prevalence of anxiety disorders among youth, and their impairments in functioning and negative mental health sequelae, there are few specialised clinical practice guidelines for the treatment of anxiety disorders in children and adolescents. ${ }^{22}$ Currently, there are ongoing debates about the delivery formats for psychotherapy, especially for CBT. The first issue is whether cognitive maturity is required for successful engagement in cognitive-behavioural treatment in young child patients, despite the fact that one study reported that CBT showed a positive effect in anxiety disorder in children younger than 7 years. ${ }^{23}$ The second issue is whether patients benefit from the involvement of parents/family at all or, if so, whether conjointly or separately. One meta-analysis of CBT reported no differences in effect sizes between trials which included and excluded parents' involvement in treatment. ${ }^{24}$ In contrast, another meta-analysis of the involvement of parents in childhood psychotherapy demonstrated that parent participation was beneficial with small effect size $(\mathrm{SMD}=0.27) .{ }^{25}$ The third issue is whether internet-based psychotherapy is as effective as face-to-face psychotherapy. Some recent meta-analyses found that involving computerised elements produced outcomes that were almost as favourable as clinic-based intervention, ${ }^{26}{ }^{27}$ but one previous systematic review showed that the levels of dropout and discontinuation were very high in internet-assisted psychotherapy. ${ }^{28}$ The fourth issue is whether there are differences in efficacy between psychotherapy delivered individually or in a group. Some studies reported that group and individual CBT were equally effective in reducing anxiety symptoms. ${ }^{29}{ }^{30} \mathrm{By}$ contrast, some studies demonstrated that only individual treatment produced significant improvement in anxiety symptoms in children (aged 8-14 years). ${ }^{31} \quad 32$ These unresolved questions lead to uncertainty in clinicians' decision-making in the management of anxiety disorders in children and adolescents. Furthermore, it is very difficult to resolve these issues by traditional meta-analyses. ${ }^{16} 1824$ There is almost no evidence about effect size in these patients, because of a lack of randomised controlled trials (RCTs) directly comparing different types and different deliveries of psychotherapies.

A better-designed approach utilising Bayesian network meta-analysis integrates direct evidence (from studies directly comparing interventions) with indirect evidence (information about two treatments derived via a common comparator) from multiple treatment comparisons, to estimate the interrelations across all treatments. ${ }^{33}$ In a network meta-analysis, all interventions that have been tested in RCTs can be simultaneously compared, and their effects can be estimated relative to each other and to a common reference condition (eg, waitlist group). This methodology can provide a hierarchical evidence to guide clinical practice. Examples include network meta-analyses of psychological and pharmacological interventions for social anxiety disorder in adults ${ }^{34}$ and of the efficacy and acceptability of psychotherapies for depression in children and adolescents. ${ }^{35}$ Owing to the lack of practical information and evidence on anxiety disorder treatment in children and adolescents, we urgently need outcomes from welldesigned meta-analyses and especially from network meta-analyses. Therefore, the main aim of the present research was to compare the efficacy and acceptability of different psychotherapies in the treatment of anxiety disorders in children and adolescents.

\section{METHODS}

\section{Criteria for included studies}

\section{Types of studies}

Any RCTs, including crossover trials and clusterrandomised trials, will be included. We will only consider the results from the first randomisation period when a trial uses a crossover design. We will exclude trials in which the duration of treatment is less than 6 weeks, and trials in which the number of sessions is less than six sessions. We will also exclude trials in which the number of participants is less than 10 .

\section{Types of participants}

We will include children and adolescents (aged less than 18 when they initially enrolled in the studies) with a 
primary diagnosis of a current anxiety disorder according to standardised diagnostic criteria, for example, the Diagnostic and Statistical Manual of Mental Disorders (DSM) ${ }^{36-40}$ the International Classification of Diseases (ICD) ${ }^{41}{ }^{42}$ According to DMS-5, ${ }^{40}$ anxiety disorder includes separation anxiety disorder, selective mutism, specific phobia, social anxiety disorder (social phobia), panic disorder, agoraphobia, GAD and panic attack specifier. Where studies include 'mixed disorders', we will also exclude trials involving more than $20 \%$ patients with a primary diagnosis of PTSD and/or OCD in this network meta-analysis. Where trials include both adults and children/adolescents, the data will be included if data in youths can be extracted separately, or obtained from authors. We will also consider including trials in which participants have a secondary diagnosis of comorbid general psychiatric disorders, for example, major depression, attention deficit hyperactivity disorder and autism spectrum disorder. However, we will exclude studies in which participants are described as only having anxiety symptoms. All research settings, such as outpatient clinics, inpatient services, community clinics and schools, will be included.

\section{Types of interventions}

We will include all published and unpublished RCTs comparing any psychotherapy with either the control condition or the active comparator for the acute treatment of anxiety disorders. The most common psychotherapy for anxiety disorders in children and adolescents is CBT, delivered in different intervention formats (group or individual; childhood psychotherapy, plus family/parental involvement or only family/parental therapy; and face-to-face or internet-based). There are other types of psychotherapies for anxiety disorders in children and adolescents, for example, behaviour therapy, bibliotherapy, modelling, cognitive bias modification and reinforced practice. ${ }^{43}{ }^{44}$ Since there may exist different treatment effects among different treatment types and deliveries of psychotherapies, we will view them as independent nodes in the network analysis. Also, in principle, any patient who meets all inclusion criteria is equally likely to be randomised to any of the interventions in the synthesis comparator set in the network meta-analysis. ${ }^{45}$ Thus, we will exclude the intervention for specific anxiety disorder, such as one session treatment (in the exposure in vivo group) for specific phobia.

In terms of control conditions, waiting list (WL), non-treatment (NT), treatment as usual (TAU) and psychological placebo (PBO) will be included. TAU is considered to be an unstructured psychological intervention that may have some treatment effects (eg, usual care, health education class or traditional counselling) in some settings, and for some clinicians, TAU may include some elements of evidence-based treatments, whereas NT and WL control can be considered not to have any active treatment components. PBO is a control condition which is regarded as inactive on improving anxiety symptoms by the researchers, but involves time spent with the participants, such as regular reviews of mental health symptoms and interventions, supportive counselling, problem discussions and psychodrama. Owing to the different treatment effects among these control conditions as reported in previous meta-analyses, we will view these control conditions as independent nodes in this network meta-analysis.

Trials in which psychotherapy is used as a combination strategy (eg, combining different psychotherapies or psychotherapy and medication) and as an augmentation strategy will be excluded, because such designs make it impossible to detect effects associated with each specific treatment approach. Trials designed as maintenance treatment or relapse prevention will be excluded. We will also exclude trials in which the psychotherapy intervention was not designed to specifically treat anxiety disorders. We will exclude the trials with drug placebo control, because psychological and drug placebo are different on multiple dimensions. Studies with concomitant use of an auxiliary psychotropic agent (eg, benzodiazepine) will be also excluded.

\section{Types of outcome measures}

We will assess efficacy at post-treatment and at follow-up, in order to examine the effects of acute phase treatment and longer term effects. The standardised mean difference (SMD) was used as a measure of effect size in efficacy outcome. It was calculated as the difference between the mean change scores of the two groups divided by the pooled SD. The mean change scores equal end point minus baseline scores for two groups. The primary outcome will be efficacy at post-treatment, as measured by mean change scores in anxiety symptoms from baseline to post-treatment. The secondary outcome measure will be efficacy at follow-up, as measured by mean change scores in anxiety symptoms from baseline to the end of full follow-up. Where available, we will extract data at short-term (1-6 months) follow-up and long-term (6-12 months) follow-up for each study, and at the longest available follow-up. We will not include participants in the follow-up analysis who take part in the follow-up treatment (eg, continuous treatment or booster sessions).

Anxiety symptoms will be measured using various psychometrically continuous scales, including (1) Revised Children's Manifest Anxiety Scale (RCMAS) (Reynolds 1985), ${ }^{46}$ (2) Spence Child Anxiety Scale, Child and Parent Versions (SCAS) ${ }^{47}$ (3) Screen for Child Anxiety-Related Emotional Disorders (SCARED), ${ }^{48}$ (4) Fear Survey for Children Revised (FSSC-R), ${ }^{49}$ (5) Social Phobia and Anxiety Inventory for Children (SPAI-C) ${ }^{50}$ and (6) Child Behaviour Checklist (CBCL) ${ }^{51}$ Where anxiety symptoms are measured using more than one continuous scale in a trial, we will extract data on the basis of a hierarchy of anxiety rating scales, based on psychometric properties and frequency for use with 
children and adolescents (please see table 1). We will also establish a hierarchy of informants of anxiety rating scales, with the child or adolescent self-report first in the hierarchy, then the parent/teacher report and then the clinician report.

In addition, the acceptability of treatment will be defined as all-cause discontinuation, as measured by the proportion of patients who discontinued treatment for any reason during the acute phase treatment. The acceptability encompasses efficacy and tolerability in previous meta-analyses, ${ }^{35} 365253$ but clinicians might intuitively associate the word acceptability more with tolerability than with efficacy. However, in psychotherapy trials, acceptability may be more connected with treatment efficacy than tolerability, because few adverse effects are reported in these psychotherapy trials; in addition, acceptability may also be influenced by the views and concerns of the youths and/or their parents.

We will also examine continuous measures of quality of life and functional improvement (QoL/functioning).$^{5455}$ The scales of quality of life include the Quality of Life Inventory (QoLI), ${ }^{56}$ Pediatric Quality of Life Inventory (QoL Child Report), ${ }^{57}$ etc. The scales of functional improvement include the Children's Global Assessment Scale (CGAS), ${ }^{58}$ the Sheehan Disability Scale (SDS), ${ }^{59}$ etc. When data are reported on the quality of life and functional improvement, we will first choose data from the quality of life.

Table 1 Hierarchy of anxiety symptom severity measurement scales

\begin{tabular}{|c|c|c|}
\hline Hierarchy & $\begin{array}{l}\text { Anxiety symptom severity } \\
\text { rating scales }\end{array}$ & Abbreviation \\
\hline 1 & $\begin{array}{l}\text { Revised Children's Manifest } \\
\text { Anxiety Scale }\end{array}$ & RCMAS \\
\hline 2 & $\begin{array}{l}\text { Spence Children's Anxiety } \\
\text { Scale }\end{array}$ & SCAS \\
\hline 3 & $\begin{array}{l}\text { Multidimensional Anxiety } \\
\text { Scale for Children }\end{array}$ & MASC \\
\hline 4 & $\begin{array}{l}\text { State-Trait Anxiety Inventory } \\
\text { for Children }\end{array}$ & STAIC \\
\hline 5 & $\begin{array}{l}\text { Screen for Anxiety and } \\
\text { Related Disorders }\end{array}$ & SCARED \\
\hline 6 & $\begin{array}{l}\text { Revised Child Anxiety and } \\
\text { Depression Scale }\end{array}$ & RCADS \\
\hline 7 & Clinician severity ratings & CSR \\
\hline 8 & $\begin{array}{l}\text { Fear Survey Schedule for } \\
\text { Children }\end{array}$ & FSSC \\
\hline 9 & $\begin{array}{l}\text { Childhood Anxiety Sensitivity } \\
\text { Index }\end{array}$ & CASI \\
\hline 10 & Beck Anxiety Inventory & $\mathrm{BAl}$ \\
\hline 11 & $\begin{array}{l}\text { Child Behavior } \\
\text { Checklist-Internalising }\end{array}$ & CBCL-Int \\
\hline 12 & Pediatric Anxiety Rating Scale & PARS \\
\hline
\end{tabular}

Where different anxiety symptom severity rating scales were used for the purpose of pooling results, we chose the single best available outcome measure according to a hierarchy based on psychometric properties and frequency of use with children and adolescents.

\section{Search strategy}

We will identify relevant published and unpublished trials from systematic searches in the following electronic databases: PubMed, EMBASE, Cochrane, Web of Science, PsycINFO, CINAHL, ProQuest Dissertations and LiLACS. The search time frame will be from 1 January 1966 to 30 January 2015 (update to 1 July 2015). There will be no restrictions on language, publication year or publication type. We will search these databases using the following search strategy with text words and subject headings. The text words including: Condition $=($ anxiety OR anxious OR phobic OR fear OR phobia OR "panic disorder*" OR "overanxious disorder*" OR "avoidant disorder*" OR agoraphobia OR "selective mutism" OR "panic attack specifier" OR "combat disorder*" OR "mixed disorder*" OR neurosis OR neuroses OR neurotic OR "school refusal") AND Intervention $=($ psychother* OR behavio* OR "family therap*" OR "family treatment" OR cogniti* OR interpersonal OR relaxation OR bibliotherap* OR counsel* OR supportive OR problem-solving OR "problem solving" OR psychodynamic OR Modeling OR "reinforced practice" OR exposure OR treatment OR treatments OR therapy) AND Age $=($ adolesc* OR child* OR boy* OR girl* OR juvenil* OR minors OR paediatri* OR pediatri* OR pubescen* OR school* OR student* OR teen* OR young OR youth* OR class*). The subject headings including: "Anxiety Disorders" AND "Psychotherapy" AND ("child" OR "adolescent"). We will also search clinicaltrials.gov in the USA, the Australian New Zealand Clinical Trials Registry (ANZCTR) and the Chinese Clinical Trial Register (ChiCTR) for ongoing trial registers, and check relevant reports on the U.S. Food and Drug Administration (FDA) website, the International Clinical Trials Registry Platform (ICTRP) in the $\mathrm{WHO}$, and manually search major psychiatric and psychology journals, such as $J$ Anxiety Disord, J Child Adolesc Psychopharmacol, J Clin Child Adolesc Psychol, Clin Psychol Rev, J Child Psychol Psychiatry, Clin Child Psychol Psychiatry, J Am Acad Child Adolesc Psychiatry, J Consult Clin Psychol, Cognit Ther Res, Psychopharmacol Bull, Psychother Psychosom, Arch Gen Psychiatry, Am J Psychiatry, Depress Anxiety. Additional relevant studies will be obtained by scanning reference lists of trials identified in the initial searches and relevant meta-analyses or systematic reviews. ${ }^{16-18}$ In addition, all relevant authors, including some authors of included trials and of some important evidence-based papers, will be contacted to supplement incomplete information. The flow chart form will be used to present the search strategy used in this meta-analysis.

\section{Study selection and data extraction Selection of trials}

Titles and abstracts identified from the search strategies will be independently examined by two reviewers (BQ and $\mathrm{YZ}$ ). If both reviewers judge that the trial does not meet the eligibility criteria, we will exclude it. Then we 
will obtain the full texts of all remaining articles and determine whether to include them by the same eligibility criteria. We will calculate the inter-rater reliability of the two raters. Any disagreements will be resolved by a third review author (XZ). When multiple publications are from the same data set, we will select the trial with the most complete data. In addition, the references of relevant reviews and included trials will also be checked by $B Q$ and YZ. The reasons for exclusion of trials will be reported in the characteristics of the excluded studies list.

\section{Risk of bias assessment}

The methodological quality of the included studies will be independently assessed by two reviewers (BQ and $\mathrm{YZ}$ ). We will assess risk of bias as 'low risk', 'unclear risk' or 'high risk', in accordance with the Cochrane Collaboration's Risk of bias tool as described in the Cochrane Handbook for Systematic Reviews of Interventions. ${ }^{60}$ The following items will be assessed: sequence generation, allocation concealment, blinding of participants and personnel, blinding of outcome assessors, incomplete outcome data, selective outcome reporting and other sources of bias. The inter-rater reliability of the assessment of risk of bias of included studies will be calculated. Any disagreements will be resolved by a third review author (XZ).

\section{Data extraction}

Two independent reviewers (BQ and YL) will extract the data from the original reports using standardised data extraction forms, including study characteristics (such as first listed author, publication year, title, publication type, publication journal, country and sponsor), patient characteristics (such as diagnostic criteria, type of anxiety, level of anxiety symptoms, comorbidities, the age of patients, the number of patients and the gender of patients), intervention details (such as psychotherapy type, intervention formats, treatment conditions and different delivery modalities, the session of psychotherapy, the duration of acute treatment and follow-up and cointerventions) and outcome measures (efficacy outcomes at acute treatment and follow-up, acceptability outcome, QoL/functioning outcome). The main characteristics of trials included in this review will be presented in a table. Any disagreements will be resolved by a third review author (XZ). We will assess and report the reliability of the reviewers' data extraction.

\section{Statistical analysis}

Network meta-analysis combines direct and indirect evidence for all relative treatment effects and provides estimates with maximum power. ${ }^{61}{ }^{62}$ First, we will perform pairwise meta-analyses of direct evidence using the random-effects model with Stata V.13.0. Second, we will perform a random-effects network meta-analysis within a Bayesian framework using Markov chain Monte Carlo method in WinBUGS V.1.4.3. The pooled estimates of SMD with $95 \%$ credible intervals (CrIs) will be calculated for the continuous outcomes; and OR with 95\% CrIs will be calculated for the dichotomous outcomes. In the presence of minimally informative priors, CrIs can be interpreted similarly to CIs. Intent-to-treat (ITT) analyses will be undertaken. For symptoms, last observation carried forward (LOCF) analyses will be performed if authors supply the data.

The pooled estimates will be obtained using the Markov Chains Monte Carlo method. Two Markov chains will be run simultaneously with different arbitrarily chosen initial values. To ensure convergence, trace plots and the Brooks-Gelman-Rubin statistic will be assessed. ${ }^{63}$ Convergence will be found to be adequate after running 50000 samples for both chains. These samples will then be discarded as 'burn-in', and posterior summaries will be based on 100000 subsequent simulations. When a loop connected three treatments, it is possible to evaluate the inconsistency between direct and indirect evidence. The node splitting method will be used to calculate the inconsistency of the model, which separates evidence on a particular comparison into direct and indirect evidence. ${ }^{64}$ Probability values will be summarised and reported as surface under the cumulative ranking curve (SUCRA), a simple transformation of the mean rank used to provide a hierarchy of the treatments that accounts for both the location and the variance of all relative treatment effects. ${ }^{65}$ We will graphically present the data and evaluate inconsistency and SUCRA using computational and graphical tools with STATA V.13.0. ${ }^{66}$

\section{Subgroup analysis}

Where possible, the following subgroup analyses will be performed for the: (1) age of participants (eg, children aged 6-12 vs adolescents from 13 to 18 years); (2) duration of psychotherapy (eg, short-term treatment of 8 or fewer weeks vs long-term treatment of more than 8 weeks); (3) the number of sessions (eg, 10 or fewer sessions vs more than 10 sessions); (4) sex ratio (male-to-female ratio $>1$ vs male-to-female ratio $<1$ ); (5) sample size; (6) with comorbid general psychiatric disorders versus without comorbid general psychiatric disorders; (7) published year; and (8) the source of outcome information (self-rated vs other-rated).

\section{Other analyses}

Funnel plot analyses and Egger's test will be performed to check for publication bias. ${ }^{60}$ Moreover, we will carry out meta-regression analyses to investigate the effect of sponsorship and year published on outcome estimate.

\section{Ethics and dissemination}

This systematic review and network meta-analysis will be published in a peer-reviewed journal. It will be disseminated electronically and in print. Since no primary data collection will be undertaken, no additional formal ethical assessment and informed consent are required. 
Author affiliations

${ }^{1}$ Department of Neurology, The First Affiliated Hospital of Chongqing Medical University, Chongqing, China

${ }^{2}$ Department of Psychiatry, University of Oxford, Oxford, UK

${ }^{3}$ Highfield Family and Adolescent Unit, Warneford Hospital, Oxford, UK

${ }^{4}$ Research Department of Clinical, Educational \& Health Psychology, Centre

for Outcomes Research and Effectiveness, University College London,

London, UK

${ }^{5}$ Department of Clinical Psychology, VU University Amsterdam, Amsterdam,

The Netherlands

${ }^{6}$ Department of Diagnostic, Clinical and Public Health Medicine, University of Modena and Reggio Emilia, Modena, Italy

${ }^{7}$ Department of Child and Adolescent Psychiatry, Institut des Systèmes Intelligents et de Robotiques, UPMC Univ Paris 06, UMR 7222, Sorbonne Universités, AP-HP, Hôpital Pitié-Salpétrière, Paris, France

${ }^{8}$ Department of Psychology, Harvard University, Cambridge, Massachusetts, USA

Contributors $\mathrm{YZ}$ and $\mathrm{XZ}$ conceived the study. $\mathrm{YZ}$ and $\mathrm{PX}$ drafted the protocol manuscript. ACJ, CJW, PM, DC and JRW assisted in the protocol design and revision. $B Q$ and $Y L$ participated in the search strategy development. $X Z$ and CDG designed the statistical analysis; PX and JRW are the guarantors. All authors read and approved the final version of the manuscript.

Competing interests During the past 2 years, DC reported past consultation for or the receipt of honoraria from Bristol-Myers Squibb, Otsuka, Shire, Lundbeck and IntegraGen. He also received a grant from the Pfizer Foundation.

\section{Provenance and peer review Not commissioned; externally peer reviewed.}

Open Access This is an Open Access article distributed in accordance with the Creative Commons Attribution Non Commercial (CC BY-NC 4.0) license, which permits others to distribute, remix, adapt, build upon this work noncommercially, and license their derivative works on different terms, provided the original work is properly cited and the use is non-commercial. See: http:// creativecommons.org/licenses/by-nc/4.0/

\section{REFERENCES}

1. Costello EJ, Egger HL, Angold A. Developmental epidemiology of anxiety disorders. In: Ollendick TH, March JS, eds. Phobic and anxiety disorders in children and adolescents: a clinician's guide to effective psychosocial and pharmacological interventions. New York: Oxford University Press, 2004:61-91.

2. Essau CA, Gabbidon J. Epidemiology, comorbidity and mental health service utilization. In: Essau CA, Ollendick TH, eds. The Wiley-Blackwell handbook of the treatment of childhood and adolescent anxiety. 1st edn. Chichester: Wiley-Blackwell, 2013:23-42

3. Beesdo K, Knappe S, Pine DS. Anxiety and anxiety disorders in children and adolescents: developmental issues and implications for DSM-V. Psychiatr Clin North Am 2009;32:483-524.

4. Kendall PC, Compton SN, Walkup JT. Clinical characteristics of anxiety disordered youth. J Anxiety Disord 2010;24:360-5.

5. National Institute for Health and Clinical Excellence (NICE) Common mental health disorders- Identification and pathways to care. http://www.nice.org.uk/guidance/cg123 (accessed 3 Feb 2015)

6. Vasa RA, Carroll LM, Nozzolillo AA, et al. A systematic review of treatments for anxiety in youth with autism spectrum disorders. J Autism Dev Disord 2014;44:3215-29.

7. Kovacs M, Gatsonis C, Paulauskas SL, et al. Depressive disorders in childhood. IV. A longitudinal study of comorbidity with and risk for anxiety disorders. Arch Gen Psychiatry 1989;46:776-82.

8. Bittner A, Egger HL, Erkanli A, et al. What do childhood anxiety disorders predict? J Child Psychol Psychiatry 2007;48:1174-83.

9. Kushner MG, Sher KJ, Beitman BD. The relation between alcohol problems and the anxiety disorders. Am J Psychiatry 1990;147:685-95.

10. Hill RM, Castellanos D, Pettit JW. Suicide-related behaviors and anxiety in children and adolescents: a review. Clin Psychol Rev 2011;31:1133-44.

11. Pine DS, Helfinstein SM, Bar-Haim Y, et al. Challenges in developing novel treatments for childhood disorders: lessons from research on anxiety. Neuropsychopharmacology 2009;34:213-28.

12. Langley AK, Bergman RL, McCracken J, et al. Impairment in childhood anxiety disorders: preliminary examination of the child anxiety impact scale-parent version. J Child Adolesc Psychopharmacol 2004;14:105-14.

13. Beesdo K, Bittner A, Pine DS, et al. Incidence of social anxiety disorder and the consistent risk for secondary depression in the first three decades of life. Arch Gen Psychiatry 2007;64:903-12.

14. Kim-Cohen J, Caspi A, Moffitt TE, et al. Prior juvenile diagnoses in adults with mental disorder: developmental follow-back of a prospective-longitudinal cohort. Arch Gen Psychiatry 2003;60:709-17.

15. Creswell C, Waite P, Cooper PJ. Assessment and management of anxiety disorders in children and adolescents. Arch Dis Child 2014;99:674-8.

16. Reynolds S, Wilson C, Austin J, et al. Effects of psychotherapy for anxiety in children and adolescents: a meta-analytic review. Clin Psychol Rev 2012;32:251-62.

17. Davis TE III, May A, Whiting SE. Evidence-based treatment of anxiety and phobia in children and adolescents: current status and effects on the emotional response. Clin Psychol Rev 2011;31:592-602.

18. James AC, James G, Cowdrey FA, et al Cognitive behavioural therapy for anxiety disorders in children and adolescents. Cochrane Database Syst Rev 2013;(6):CD004690. 10.1002/14651858. CD004690.pub3

19. Kendall PC. Treating anxiety disorders in children: results of a randomized clinical trial. J Consult Clin Psychol 1994;62: $100-10$.

20. Muris P, Mayer B, den Adel M, et al. Predictors of change following cognitive-behavioral treatment of children with anxiety problems: a preliminary investigation on negative automatic thoughts and anxiety control. Child Psychiatry Hum Dev 2009;40:139-51.

21. Lau JY, Belli SR, Chopra RB. Cognitive bias modification training in adolescents reduces anxiety to a psychological challenge. Clin Child Psychol Psychiatry 2013;18:322-33.

22. Higa-McMillan CK, Francis SE, Rith-Najarian L, et al. Evidence base update: 50 years of research on treatment for child and adolescent anxiety. J Clin Child Adolesc Psychol 2015;18:1-23.

23. Hirshfeld-Becker DR, Masek B, Henin A, et al. Cognitive behavioural therapy for 4- to 7-year old children with anxiety disorders: a randomized clinical trial. J Consult Clin Psychol 2010;78: 498-510.

24. In-Albon T, Schneider S. Psychotherapy of childhood anxiety disorders: a meta-analysis. Psychother Psychosom 2007;76:15-24.

25. Dowell KA, Ogles BM. The effects of parent participation on child psychotherapy outcome: a meta-analytic review. J Clin Child Adolesc Psychol 2010;39:151-62.

26. Ye X, Bapuji SB, Winters SE, et al. Effectiveness of internet-based interventions for children, youth, and young adults with anxiety and/ or depression: a systematic review and meta-analysis. BMC Health Serv Res 2014;14:313.

27. Rooksby M, Elouafkaoui $P$, Humphris $G$, et al. Internet-assisted delivery of cognitive behavioural therapy (CBT) for childhood anxiety: systematic review and meta-analysis. $J$ Anxiety Disord 2015;29:83-92.

28. Richardson T, Stallard P, Velleman S. Computerised cognitive behavioural therapy for the prevention and treatment of depression and anxiety in children and adolescents: a systematic review. Clin Child Fam Psychol Rev 2010;13:275-90.

29. Barrett P, Healy-Farrell L, March JS. Cognitive-behavioral family treatment of childhood obsessive-compulsive disorder: a controlled trial. J Am Acad Child Adolesc Psychiatry 2004;43:46-62.

30. Muris $\mathrm{P}$, Mayer $\mathrm{B}$, Bartelds $\mathrm{E}$, et al. The revised version of the Screen for Child Anxiety Related Emotional Disorders (SCARED-R): treatment sensitivity in an early intervention trial for childhood anxiety disorders. Br J Clin Psychol 2001;40:323-36.

31. Flannery-Schroeder EC, Kendall PC. Group and individual cognitive-behavioral treatments for youth with anxiety disorders: a randomized clinical trial. Cognit Ther Res 2000;24:251-78.

32. Manassis K, Mendlowitz SL, Scapillato D, et al. Group and individua cognitive-behavioral therapy for childhood anxiety disorders: a randomized trial. J Am Acad Child Adolesc Psychiatry 2002;41:1423-30.

33. Salanti G, Higgins JP, Ades AE, et al. Evaluation of networks of randomized trials. Stat Methods Med Res 2008;17:279-301.

34. Mayo-Wilson E, Dias S, Mavranezouli I, et al. Psychological and pharmacological interventions for social anxiety disorder in adults: a systematic review and network meta-analysis. Lancet Psychiatry 2014;1:368-76.

35. Zhou X, Hetrick SE, Cuijpers $P$, et al. Comparative efficacy and acceptability of psychotherapies for depression in children and adolescents: a systematic review and network meta-analysis. World Psychiatry 2015;14:207-22. 
36. American Psychiatric Association. Diagnostic and statistical manual of mental disorders (DSM-III). 3rd edn. Washington DC: American Psychiatric Association, 1980.

37. American Psychiatric Association. Diagnostic and statisticalmanual of mental disorders (DSM-III-R). 3rd edn. Washington DC: American Psychiatric Association, 1987.

38. American Psychiatric Association. Diagnostic and statistical manual of mental disorders (DSM-IV). 4th edn. Washington DC: American Psychiatric Association, 1994.

39. American Psychiatric Association. Diagnostic and statistical manual of mental disorders (DSM-IV-TR). 4th edn. Washington DC: American Psychiatric Association, 2000.

40. American Psychiatric Association. Diagnostic and statistical manual of mental disorders. 5th edn. Arlington, VA: American Psychiatric Publishing, 2013

41. World Health Organization. The ICD-9 classification of mental and behavioural disorders: clinical description and diagnostic guidelines. Geneva: World Health Organization, 1978.

42. World Health Organization. The ICD-10 classification of mental and behavioural disorders: clinical description and diagnostic guidelines. Geneva: World Health Organization, 1992.

43. Weisz JR. Psychotherapy for children and adolescents: evidence-based treatments and case examples. Cambridge, UK: Cambridge University Press, 2004

44. Cristea IA, Mogoase C, David D, et al. Practitioner review: cognitive bias modification for mental health problems in children and adolescents: a meta-analysis. J Child Psychol Psychiatry 2015;56:723-34.

45. Filippini G, Del Giovane C, Vacchi L, et al. Immunomodulators and immunosuppressants for multiple sclerosis: a network meta-analysis. Cochrane Database Syst Rev 2013;(6):CD008933. 10.1002/ 14651858.CD008933.pub2

46. Reynolds CR, Richmond BO. Revised children's manifest anxiety scale (RCAMS) manual. Los Angeles, CA: Western Psychological Services, 1985

47. Spence S. Structure of anxiety symptoms amongst children: a confirmatory factor-analytic study. J Abnorm Psychol 1997;106:280-97.

48. Birmaher B. Screen for child anxiety related emotional disorders (SCARED). Pittsburgh, PA: Western Psychiatric Institute and Clinic, 1999.

49. Ollendick TH, King NJ. Empirically supported treatments for children with phobic and anxiety disorders: current status. J Clin Child Psychol 1998;27:156-67.

50. Beidel DC, Turner SM, Morris TL. A new instrument to assess childhood social anxiety and phobia: the Social Phobia and Anxiety Inventory for Children. Psychol Assess 1995;7:73-9.
51. Achenbach T. Program manual for the child behavior checklist 4-18 profile. Burlington, VT: University Associates in Psychiatry, 1991.

52. Leucht S, Cipriani A, Spineli L, et al. Comparative efficacy and tolerability of 15 antipsychotic drugs in schizophrenia: a multiple-treatments meta-analysis. Lancet 2013;382:951-62.

53. Stuhec M, Munda B, Svab V, et al. Comparative efficacy and acceptability of atomoxetine, lisdexamfetamine, bupropion and methylphenidate in treatment of attention deficit hyperactivity disorder in children and adolescents: a meta-analysis with focus on bupropion. J Affect Disord. 2015;178:149-59.

54. Olatunji BO, Cisler JM, Tolin DF. Quality of life in the anxiety disorders: a meta-analytic review. Clin Psychol Rev 2007;27:572-81.

55. Settipani CA, Kendall PC. Social functioning in youth with anxiety disorders: association with anxiety severity and outcomes from cognitive-behavioral therapy. Child Psychiatry Hum Dev 2013;44:1-18.

56. Frisch MB, Cornell J, Villanueva $\mathrm{M}$, et al. Clinical validation of the Quality of Life Inventory. A measure of life satisfaction for use in treatment planning and outcome assessment. Psychol Assess 1992;4:92-101.

57. Varni JW, Seid M, Rode CA. The PedsQL: measurement model fo the pediatric quality of life inventory. Med Care 1999;37:126-39.

58. Shaffer D, Gould MS, Brasic J, et al. A children's global assessment scale (CGAS). Arch Gen Psychiatry 1983;40:1228-31.

59. Sheehan DV, Harnett-Sheehan K, Raj BA. The measurement of disability. Int Clin Psychopharmacol 1996;11:89-95.

60. Higgins JPT, Green S. Cochrane handbook for systematic reviews of interventions version 5.1. 0. [updated March 2011]. The Cochrane Collaboration, 2011. http://www.cochrane- handbook.org (accessed 1 Oct 2011).

61. Lu G, Ades AE. Combination of direct and indirect evidence in mixed treatment comparisons. Stat Med 2004;23:3105-24.

62. Caldwell DM, Ades AE, Higgins JP. Simultaneous comparison of multiple treatments: combining direct and indirect evidence. BMJ 2005;331:897-900.

63. Brooks SP, Gelman A. Alternative methods for monitoring convergence of iterative simulations. J Comput Graph Stat 1998;7:434-45.

64. Lu G, Ades A. Assessing evidence inconsistency in mixed treatment comparisons. J Am Stat Assoc 2006;101:447-59.

65. Salanti G, Ades AE, loannidis JP. Graphical methods and numerical summaries for presenting results from multiple-treatment meta-analysis: an overview and tutorial. J Clin Epidemiol 2011;64:163-71.

66. Chaimani A, Higgins JP, Mavridis D, et al. Graphical tools for network meta-analysis in STATA. PLOS ONE 2013;8:e76654 